built so fuel flows smoothly and no membrane is required, but these have low power density and are usually not rechargeable.

Cullen Buie and his colleagues at the Massachusetts Institute of Technology in Cambridge engineered a membraneless hydrogenbromine battery that can pump fuels at very high concentrations. The design boosts power density to three times that reported for other membrane-free batteries, and the battery can be recharged. Nature Commun. http://doi. org/njt (2013)

\section{ATOMIC PHYSICS}

\section{Super-stable clocks}

The most stable atomic clock built so far loses or gains less than one second every quintillion seconds (equal to $10^{18}$ seconds, or more than 30 billion years), which is about ten times better than the previous record.

Andrew Ludlow at the National Institute of Standards and Technology in Boulder, Colorado, and his colleagues used lasers to monitor the oscillation of energy levels in two sets of ultracold ytterbium atoms that had been trapped using magnetic fields and laser beams. The clocks ticked in sync with a stability of about one part in $10^{18}$.

Such stable clocks could benefit satellite communication and navigation, and would enable space-based tests of general relativity to be conducted about 1,000 times more precisely than with clocks available today.

\section{Science http://doi.org/nj9} (2013)

\section{NEUROSCIENCE}

\section{Single gene tweak for prion diseases}

Engineered mouse strains develop rare prion diseases without the need for an infectious agent.

Susan Lindquist of the
Whitehead Institute for Biomedical Research in Cambridge, Massachusetts, and her colleagues have created mouse models for two fatal human diseases caused by prions - misfolded proteins that spur other proteins to misfold. Creutzfeldt-Jakob disease and fatal familial insomnia affect different parts of the brain and arise from different mutations in the same gene. Rather than inserting mutated genes into the mouse genome - a tactic that ineffectively mimics the disease - the researchers mutated the prion gene in place.

Single amino-acid changes in the mouse prion protein caused the brain degeneration that is characteristic of each disease, and both models produce prions that can infect other mice.

Proc. Natl Acad. Sci. USA http://doi.org/njv (2013)

\section{CANCER BIOLOGY}

\section{Cancer mutation has a long reach}

Some DNA mutations that promote cancer may work through extra copies not of genes, but of genetic regulatory sequences.

Duplications of the chromosomal sequences $17 q 23$ and 20 q13 predict poorer outcomes in certain breast cancers - but no genes in these regions suggest a mechanism for how. Tim Huang and co-workers at the University of Texas Health Science Center in San Antonio sequenced genomic regions that physically interact with other stretches of DNA that bind to the oestrogen receptor, a protein complex implicated in breast cancer. This identified sites within $17 \mathrm{q} 23$ and $20 \mathrm{q} 13$ called distant oestrogen response elements (DEREs), which help the genetic material to form loops that interact with far-off genomic regions. Extra DEREs promoted more looping and deregulated genes in ways expected to promote tumour growth.

Cancer Cell 24, 197-212 (2013)

COMMUNITY CHOICE

The most viewed papers in science

\title{
Whales hear the noise
}

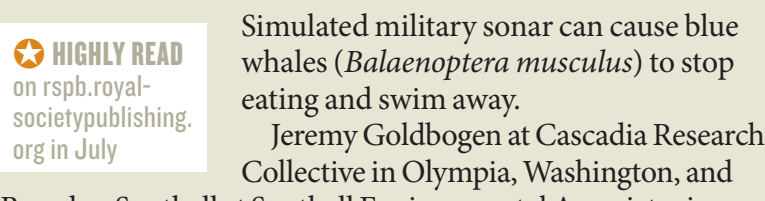

Brandon Southall at Southall Environmental Associates in Aptos, California, and their colleagues attached sensors to 17 blue whales off southern California. The team tracked the animals before, during and after playing sonar-like sounds or white noise from a research vessel. Whales at the surface typically did not respond, but whales at depth often showed behaviours such as swimming faster or ceasing to feed.

Human-produced noises could keep baleen whales from foraging and so affect their fitness, the authors say.

Proc. R. Soc. B 280, 20130657 (2013)

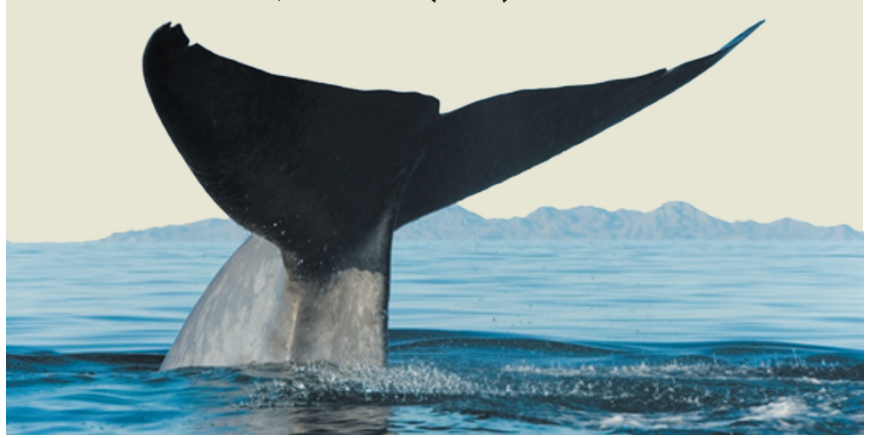

\section{MICROBIOLOGY}

\section{Gut pathogen spies others' signals}

A disease-causing bacterium 'eavesdrops' on another, usually harmless, bacterial species to help it survive antibiotics.

Under certain conditions, populations of Escherichia coli that live in the gut secrete indole, a signalling molecule that makes them more tolerant of antibiotics.

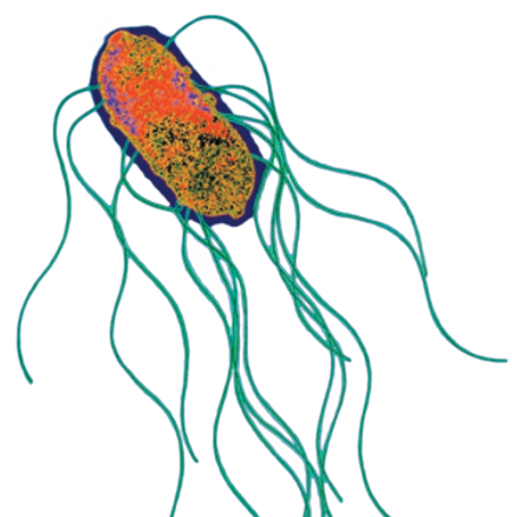

Although the gut pathogen Salmonella typhimurium (pictured) cannot produce indole, it still responds to it. James Collins at Boston University, Massachusetts, and his colleagues showed that S. typhimurium exposed to indole or to indole-producing E. coli survived better under antibiotics than did bacteria in indole-free conditions. In both species, indole strongly boosted the expression of stress-response genes known to help bacteria withstand drugs and assaults by the human immune system.

Such interactions between species could help harmful bacterial infections to persist, the authors say.

Proc. Natl Acad. Sci. USA http://doi.org/njw (2013)

\section{$\rightarrow$ NATURE,COM}

For the latest research published by Naturevisit:

www.nature.com/latestresearch 